\title{
Decomposition-Based Tracking Control with Particles
}

\author{
Mark Graham and Jules Garrett
}

\begin{abstract}
In this paper, a new control scheme, called additive-state-decomposition-based tracking control, is proposed to solve the tracking (rejection) problem for rotational position of the TORA (a nonlinear nonminimum phase system). By the additive state decomposition, the tracking (rejection) task for the considered nonlinear system is decomposed into two independent subtasks: a tracking (rejection) subtask for a linear time invariant (LTI) system, leaving a stabilization subtask for a derived nonlinear system. By the decomposition, the proposed tracking control scheme avoids solving regulation equations and can tackle the tracking (rejection) problem in the presence of any external signal (except for the frequencies at \pm 1 ) generated by a marginally stable autonomous LTI system. To demonstrate the effectiveness, numerical simulation is given.
\end{abstract}

TORA, RTAC, Nonminimum phase, Additive state decomposition.

\section{INTRODUCTION}

The tracking (rejection) problem for a nonlinear benchmark system called translational oscillator with a rotational actuator (TORA) and also known as rotational-translational actuator (RTAC) has received a considerable amount of attention these years [1]-[8]. Some results were presented concerning the tracking (rejection) problem for general external signals [2],[3]. However, the proposed control methods cannot achieve asymptotic disturbance rejection. Taking this into account, the nonlinear output regulation theory was applied to track (reject) external signals generated by an autonomous system. In this case, asymptotic disturbance rejection can be achieved. By using different measurement, the tracking (rejection) problem for translational displacement of the TORA were investigated [4]-[6]. Readers can refer to [6] for details. Based on the same benchmark system, some other work was also presented concerning the tracking (rejection) problem for rotational position by nonlinear output regulation theory [7],[8]. For the two types of tracking (rejection) problems, regulator 
equations have to be solved and then the resulting solutions will be further used in the controller design. However, the difficulty of constructing and solving regulator equations will increase as the complexity of external signals increases. Moreover, it may fail to design a controller if regulator equations have no solutions. These are our major motivation.

In this paper, the tracking (rejection) problem for rotational position of the TORA as [7],[8] is revisited by a new control scheme called additive-state-decomposition-based tracking control, which is based on the additive state decomposition ${ }^{1}$. The proposed additive state decomposition is a new decomposition manner different from the lower-order subsystem decomposition methods. Concretely, taking the system $\dot{x}(t)=f(t, x), x \in \mathbb{R}^{n}$ for example, it is decomposed into two subsystems: $\dot{x}_{1}(t)=f_{1}\left(t, x_{1}, x_{2}\right)$ and $\dot{x}_{2}(t)=f_{2}\left(t, x_{1}, x_{2}\right)$, where $x_{1} \in \mathbb{R}^{n_{1}}$ and $x_{2} \in \mathbb{R}^{n_{2}}$, respectively. The lower-order subsystem decomposition satisfies

$$
n=n_{1}+n_{2} \text { and } x=x_{1} \oplus x_{2} .
$$

By contrast, the proposed additive state decomposition satisfies

$$
n=n_{1}=n_{2} \text { and } x=x_{1}+x_{2} .
$$

In our opinion, lower-order subsystem decomposition aims to reduce the complexity of the system itself, while the additive state decomposition emphasizes the reduction of the complexity of tasks for the system.

By following the philosophy above, the original tracking (rejection) task is 'additively' decomposed into two independent subtasks, namely the tracking (rejection) subtask for a linear time invariant (LTI) system and the stabilization subtask for a derived nonlinear system. Since tracking (rejection) subtask only needs to be achieved on an LTI system, the complexity of external signals can be handled easier by the transfer function method. It is proved that the designed controller can tackle the tracking (rejection) problem for rotational position of the TORA in the presence of any external signal (except for the frequency at \pm 1 ) generated by a marginally stable autonomous LTI system.

This paper is organized as follows. In Section 2, the problem is formulated and the additive state decomposition is recalled briefly first. In Section 3, an observer is proposed to compensate for nonlinearity; then the resulting system is 'additively' decomposed into two subsystems; sequently, controllers are designed for them. In Section 4, numerical simulation is given. Section 5 concludes this paper.

\footnotetext{
${ }^{1}$ In this paper we have replaced the term "additive decomposition" in [9] with the more descriptive term "additive state decomposition".
} 


\section{Nonlinear Benchmark Problem and Additive State Decomposition}

\section{A. Nonlinear Benchmark Problem}

As shown in Fig.1, the TORA system consists of a cart attached to a wall with a spring. The cart is affected by a disturbance force $F$. An unbalanced point mass rotates around the axis in the center of the cart, which is actuated by a control torque $N$. The translational displacement of the cart is denoted by $x_{c}$ and the rotational position of the unbalanced point mass is denoted by $\theta$.

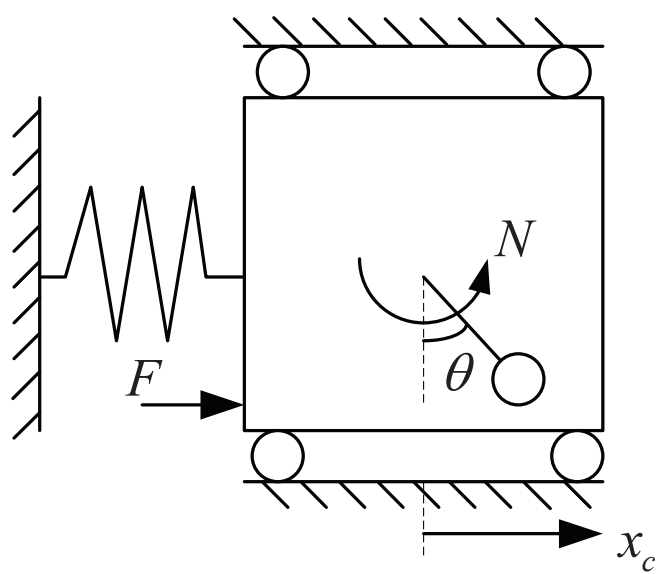

Fig. 1. TORA system configuration [1]

For simplicity, after normalization and transformation, the TORA system is described by the following state-space representation [1]:

$$
\begin{aligned}
& \dot{x}_{1}=x_{2} \\
& \dot{x}_{2}=-x_{1}+\varepsilon \sin x_{3}+F_{d} \\
& \dot{x}_{3}=x_{4} \\
& \dot{x}_{4}=u-\frac{\varepsilon \cos x_{3}}{1-\varepsilon^{2} \cos ^{2} x_{3}} F_{d}, x(0)=x_{0}
\end{aligned}
$$

where $0<\varepsilon<1, x=\left[\begin{array}{llll}x_{1} & x_{2} & x_{3} & x_{4}\end{array}\right]^{T} \in \mathbb{R}^{4}, x_{3}=\theta, F_{d} \in \mathbb{R}$ is the unknown dimensionless disturbance, $u \in \mathbb{R}$ is the dimensionless control torque. In this paper, the tracking (rejection) problem for rotational position of the TORA as [7],[8] is revisited. Concretely, for system (1), it is to design a controller $u$ such that the output $y(t)=x_{3}(t) \rightarrow r$ as $t \rightarrow \infty$, meanwhile keeping the other states bounded, where $r \in(-\pi / 2, \pi / 2)$ is a known constant. Obviously, this is a nonlinear nonminimum phase tracking problem, or 
say a nonlinear weakly minimum phase tracking problem. For system (1), the following assumptions are imposed.

Assumption 1. The state $x$ can be obtained.

Assumption 2. The disturbance $F_{d} \in \mathbb{R}$ is generated by an autonomous LTI system

$$
\dot{w}=S w, F_{d}=C_{d}^{T} w
$$

where $S=-S^{T} \in \mathbb{R}^{m \times m}, C_{d} \in \mathbb{R}^{m}$ are constant matrix, $w \in \mathbb{R}^{m}$, and the pair $\left(C_{d}^{T}, S\right)$ is observable.

Remark 1. If all eigenvalues of $S$ have zero real part, then, in suitable coordinates, the matrix $S$ can always be written to be a skew-symmetric matrix. The matrix $S$ in previous literature on the output regulation problem is often chosen in a simple form $S=\left[\begin{array}{cc}0 & \omega \\ -\omega & 0\end{array}\right]$, where $\omega$ is a positive real [4]-[8]. In such a case, $F_{d}$ is in the form as $\sin ( \pm \omega t)$ and the solution to the regulator equation is easier to obtain. However, this is a difficulty when $S$ is complicated.

\section{B. Additive State Decomposition}

In order to make the paper self-contained, the additive state decomposition [?] is recalled here briefly. Consider the following 'original' system:

$$
f(t, \dot{x}, x)=0, x(0)=x_{0}
$$

where $x \in \mathbb{R}^{n}$. We first bring in a 'primary' system having the same dimension as (3), according to:

$$
f_{p}\left(t, \dot{x}_{p}, x_{p}\right)=0, x_{p}(0)=x_{p, 0}
$$

where $x_{p} \in \mathbb{R}^{n}$. From the original system (3) and the primary system (4) we derive the following 'secondary' system:

$$
f(t, \dot{x}, x)-f_{p}\left(t, \dot{x}_{p}, x_{p}\right)=0, x(0)=x_{0}
$$

where $x_{p} \in \mathbb{R}^{n}$ is given by the primary system (4). Define a new variable $x_{s} \in \mathbb{R}^{n}$ as follows:

$$
x_{s} \triangleq x-x_{p}
$$

Then the secondary system (5) can be further written as follows:

$$
f\left(t, \dot{x}_{s}+\dot{x}_{p}, x_{s}+x_{p}\right)-f_{p}\left(t, \dot{x}_{p}, x_{p}\right)=0, x_{s}(0)=x_{0}-x_{p, 0} .
$$


From the definition (6), we have

$$
x(t)=x_{p}(t)+x_{s}(t), t \geq 0 .
$$

Remark 2. By the additive state decomposition, the system (3) is decomposed into two subsystems with the same dimension as the original system. In this sense our decomposition is "additive". In addition, this decomposition is with respect to state. So, we call it "additive state decomposition".

As a special case of (3), a class of differential dynamic systems is considered as follows:

$$
\begin{aligned}
& \dot{x}=f(t, x), x(0)=x_{0}, \\
& y=h(t, x)
\end{aligned}
$$

where $x \in \mathbb{R}^{n}$ and $y \in \mathbb{R}^{m}$. Two systems, denoted by the primary system and (derived) secondary system respectively, are defined as follows:

$$
\begin{aligned}
& \dot{x}_{p}=f_{p}\left(t, x_{p}\right), x_{p}(0)=x_{p, 0} \\
& y_{p}=h_{p}\left(t, x_{p}\right)
\end{aligned}
$$

and

$$
\begin{aligned}
& \dot{x}_{s}=f\left(t, x_{p}+x_{s}\right)-f_{p}\left(t, x_{p}\right), x_{s}(0)=x_{0}-x_{p, 0}, \\
& y_{s}=h\left(t, x_{p}+x_{s}\right)-h_{p}\left(t, x_{p}\right)
\end{aligned}
$$

where $x_{s} \triangleq x-x_{p}$ and $y_{s} \triangleq y-y_{p}$. The secondary system (11) is determined by the original system (9) and the primary system (10). From the definition, we have

$$
x(t)=x_{p}(t)+x_{s}(t), y(t)=y_{p}(t)+y_{s}(t), t \geq 0 .
$$

\section{Additive-State-Decomposition-BASED Tracking Control}

In this section, in order to decrease nonlinearity, an observer is proposed to compensate for the nonlinear term $\frac{\varepsilon \cos x_{3}}{1-\varepsilon^{2} \cos ^{2} x_{3}} F_{d}$. After the compensation, the resulting nonlinear nonminimum phase tracking system is decomposed into two systems by the additive state decomposition: an LTI system including all external signals as the primary system, leaving the secondary system with a zero equilibrium point. Therefore, the tracking problem for the original system is correspondingly decomposed into two subproblems by the additive state decomposition: a tracking problem for the LTI 'primary' system and a stabilization problem for the secondary system. Obviously, the two subproblems are easier than the original one. Therefore, the original tracking problem is simplified. The structure of the closed-loop system is shown in Fig.2. 


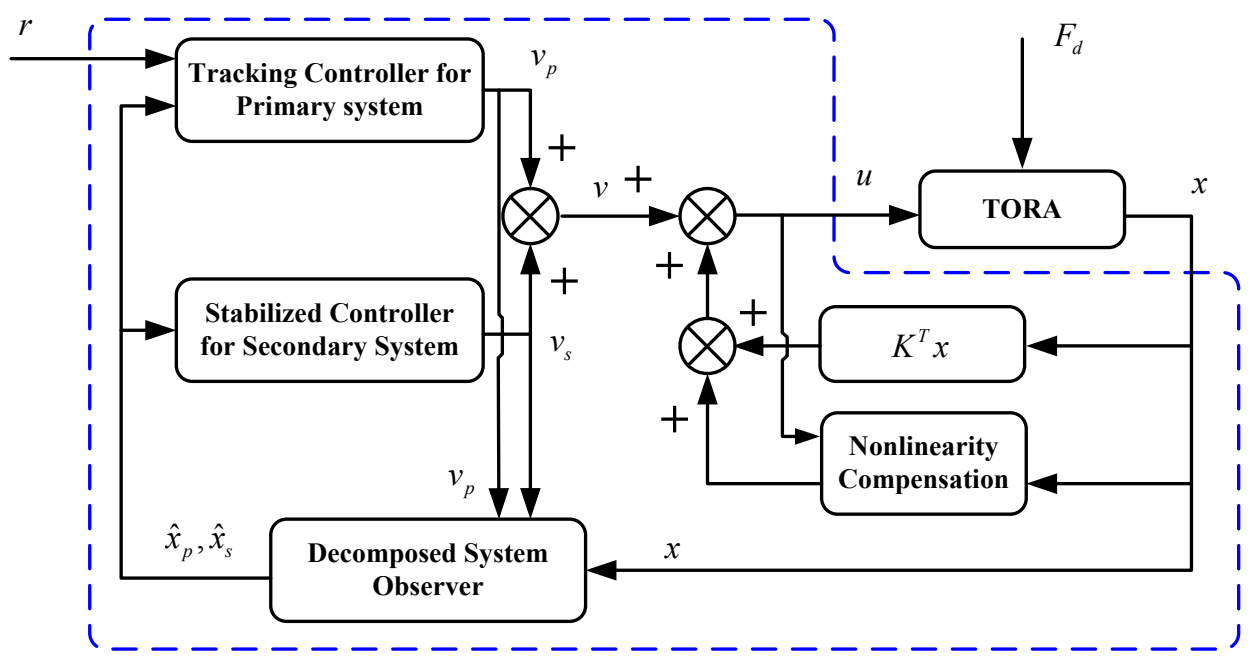

Fig. 2. Structure of the closed-loop system

\section{A. Nonlinearity Compensation}

First, in order to estimate the term $\frac{\varepsilon \cos x_{3}}{1-\varepsilon^{2} \cos ^{2} x_{3}} F_{d}$, an observer is designed, which is stated in Theorem 1.

Theorem 1. Under Assumptions 1-2, for system (1), let the observer be designed as follows

$$
\begin{aligned}
& \dot{\hat{w}}=S \hat{w}+l_{1} \frac{\varepsilon \cos x_{3}}{1-\varepsilon^{2} \cos ^{2} x_{3}} C_{d}\left(\hat{x}_{4}-x_{4}\right) \\
& \dot{\hat{x}}_{4}=-l_{2}\left(\hat{x}_{4}-x_{4}\right)-l_{1} \frac{\varepsilon \cos x_{3}}{1-\varepsilon^{2} \cos ^{2} x_{3}} C_{d}^{T} \hat{w}+u \\
& \hat{F}_{d}=l_{1} C_{d}^{T} \hat{w}, \hat{w}(0)=0, \hat{x}_{4}(0)=0
\end{aligned}
$$

where $l_{1}, l_{2}>0$. Then $\lim _{t \rightarrow \infty} \frac{\varepsilon \cos x_{3}}{1-\varepsilon^{2} \cos ^{2} x_{3}} \tilde{F}_{d}(t)=0$, where $\tilde{F}_{d} \triangleq \hat{F}_{d}-F_{d}$.

Proof. See Appendix A.

By using the observer (13), the controller $u$ in (1) is designed as follows

$$
u=K^{T} x+v+\frac{\varepsilon \cos x_{3}}{1-\varepsilon^{2} \cos ^{2} x_{3}} \hat{F}_{d}
$$

where $K \in \mathbb{R}^{4}$ and $v \in \mathbb{R}$ will be specified later. Then the system (1) becomes

$$
\begin{aligned}
& \dot{x}_{1}=x_{2} \\
& \dot{x}_{2}=-x_{1}+\varepsilon \sin x_{3}+F_{d} \\
& \dot{x}_{3}=x_{4} \\
& \dot{x}_{4}=K^{T} x+v+\frac{\varepsilon \cos x_{3}}{1-\varepsilon^{2} \cos ^{2} x_{3}} \tilde{F}_{d}, x(0)=x_{0} .
\end{aligned}
$$




\section{B. Additive State Decomposition of Original System}

Introduce a zero term $\varepsilon D(C+a B)^{T} x-\varepsilon(y+a \dot{y})=0$ into the system (14), where $a>0$, $B=\left[\begin{array}{llll}0 & 0 & 0 & 1\end{array}\right]^{T}, C=\left[\begin{array}{llll}0 & 0 & 1 & 0\end{array}\right]^{T}$ and $(C+a B)^{T} x=y+a \dot{y}$. Then the system (14) becomes

$$
\begin{aligned}
& \dot{x}=A x+B v+\phi(y, \dot{y})+D F_{d}+\varphi \\
& y=C^{T} x, x(0)=x_{0}
\end{aligned}
$$

where

$$
\begin{aligned}
A_{0} & =\left[\begin{array}{cccc}
0 & 1 & 0 & 0 \\
-1 & 0 & 0 & 0 \\
0 & 0 & 0 & 1 \\
0 & 0 & 0 & 0
\end{array}\right], A=A_{0}+B K^{T}+\varepsilon D(C+a B)^{T} \\
D & =\left[\begin{array}{l}
0 \\
1 \\
0 \\
0
\end{array}\right], \phi(y, \dot{y})=\left[\begin{array}{c}
\varepsilon \sin y-\varepsilon(y+a \dot{y}) \\
0 \\
0
\end{array}\right], \varphi=\left[\begin{array}{c}
0 \\
0 \\
0 \\
\frac{\varepsilon \cos _{3} \tilde{F}^{2} \tilde{F}_{d}}{1-\varepsilon^{2} \cos ^{2} x_{3}}
\end{array}\right] .
\end{aligned}
$$

The additive state decomposition is ready to apply to the system (15), for which the primary system is chosen to be an LTI system including all external signals as follows

$$
\begin{aligned}
& \dot{x}_{p}=A x_{p}+B v_{p}+d+\varphi \\
& y_{p}=C^{T} x_{p}, x_{p}(0)=x_{0}
\end{aligned}
$$

where $d=\phi(r, 0)+D F_{d}$. Then, according to the rule (11), the secondary system is derived from the original system (15) and the primary system (17) as follows

$$
\begin{aligned}
& \dot{x}_{s}=A x_{s}+B v_{s}+\phi\left(y_{p}+y_{s}, \dot{y}_{p}+\dot{y}_{s}\right)-\phi(r, 0) \\
& y_{s}=C^{T} x_{s}, x_{s}(0)=0
\end{aligned}
$$

where $v_{s}=v-v_{p}$. According to (12), we have

$$
x=x_{p}+x_{s} \text { and } y=y_{p}+y_{s} .
$$

Remark 3. The pair $\left(A_{0}, B\right)$ is uncontrollable, while the pair $\left(A_{0}+\varepsilon D(C+a B)^{T}, B\right)$ is controllable. Therefore, there always exists a vector $K$ such that $A=A_{0}+B K^{T}+$ $\varepsilon D(C+a B)^{T}$ is a stable matrix.

Remark 4. If $y_{p} \equiv r$ and $\dot{y}_{p} \equiv 0$, then $\left(x_{s}, v_{s}\right)=0$ is a zero equilibrium point of the secondary system (18). 
So far, the nonlinear nonminimum phase tracking system (15) is decomposed into two systems by the additive state decomposition, where the external signal $d+\varphi$ is shifted to (17) and the nonlinear term $\phi(\cdot)$ is shifted to (18). The strategy here is to assign the tracking (rejection) task to the primary system (17) and stabilization task to the secondary system (18). More concretely, in (17) design $v_{p}$ to track $r$, and design $v_{s}$ to stabilize (18). If so, by the relationship (19), $y$ can track $r$. In the following, controllers $v_{p}$ and $v_{s}$ are designed separately.

\section{Tracking Controller Design for Primary System}

Before proceeding further, we have the following preliminary result.

Consider the following linear system

$$
\begin{aligned}
& \dot{z}_{1}=S_{z} z_{1}+A_{12} e_{z} \\
& \dot{z}_{2}=A_{21} z_{1}+A_{22} z_{2}+d_{1}+\varphi_{1} \\
& e_{z}=C_{e}^{T} z_{2}+d_{2}+\varphi_{2}, z(0)=z_{0}
\end{aligned}
$$

where $S_{z} \in \mathbb{R}^{m_{1} \times m_{1}}$ is a marginally stable matrix, $A_{12} \in \mathbb{R}^{m_{1}}, C_{e} \in \mathbb{R}^{m_{2}}, A_{21} \in \mathbb{R}^{m_{2} \times m_{2}}$, $A_{22} \in \mathbb{R}^{m_{2} \times m_{1}}, z_{1} \in \mathbb{R}^{m_{1}}, z_{2}, d_{1}, \varphi_{1} \in \mathbb{R}^{m_{2}}, z=\left[\begin{array}{cc}z_{1}^{T} & z_{2}^{T}\end{array}\right]^{T} \in \mathbb{R}^{m_{1}+m_{2}}$ and $e_{z}, d_{2}, \varphi_{2} \in \mathbb{R}$.

Lemma 1. Suppose i) $\varphi_{i}(t)$ is bounded on $[0, \infty)$ and $\lim _{t \rightarrow \infty}\left\|\varphi_{i}(t)\right\|=0, i=1,2$, ii) every element of $d_{1}(t), d_{2}(t)$ are bounded on $[0, \infty)$ and can be generated by $\dot{w}_{z}=S_{z} w_{z}$, $d_{z}=C_{z}^{T} w_{z}$ with appropriate initial values, where $C_{z} \in \mathbb{R}^{m_{1}}$, iii) the parameters in (20) satisfy

$$
\max \operatorname{Re} \lambda\left(A_{z}\right)<0, A_{z}=\left[\begin{array}{cc}
S_{z} & A_{12} C_{e}^{T} \\
A_{21} & A_{22}
\end{array}\right] .
$$

Then in (20) $\lim _{t \rightarrow \infty} e_{z}(t)=0$, meanwhile keeping $z_{1}(t)$ and $z_{2}(t)$ bounded.

Proof. See Appendix B.

Define a filtered tracking error to be

$$
e_{p}=\tilde{y}_{p}+a \dot{\tilde{y}}_{p}=(C+a B)^{T} x_{p}-r
$$

where $\tilde{y}_{p}=y_{p}-r, \dot{r}=0$ and $a>0$. Let us consider the tracking problem for the primary system (17). With Lemma 1 in hand, the design of $v_{p}$ is stated in Theorem 2.

Theorem 2. For the primary system (17), let the controller $v_{p}$ be designed as follows

$$
\begin{array}{r}
\dot{\xi}=S_{a} \xi+L_{1} e_{p} \\
v_{p}\left(\xi, x_{p}, r\right)=L_{2}^{T} x_{p}+L_{3}^{T} \xi
\end{array}
$$


where $S_{a}=\operatorname{diag}(0, S), L_{1} \in \mathbb{R}^{m+1}, L_{2} \in \mathbb{R}^{4}$ and $L_{3} \in \mathbb{R}^{m+1}$ satisfy

$$
\max \operatorname{Re} \lambda\left(A_{a}\right)<0, A_{a}=\left[\begin{array}{cc}
S_{a} & L_{1}(C+a B)^{T} \\
B L_{3}^{T} & A+B L_{2}^{T}
\end{array}\right] .
$$

Then $\lim _{t \rightarrow \infty} y_{p}(t)=r$ and $\lim _{t \rightarrow \infty} \dot{y}_{p}(t)=0$ meanwhile keeping $x_{p}(t)$ and $\xi(t)$ bounded.

Proof. Incorporating the controller (23) into the primary system (17) results in

$$
\begin{aligned}
\dot{\xi} & =S_{a} \xi+L_{1} e_{p} \\
\dot{x}_{p} & =\left(A+B L_{2}^{T}\right) x_{p}+B L_{3}^{T} \xi+d+\varphi \\
e_{p} & =(C+a B)^{T} x_{p}-r
\end{aligned}
$$

where the definition (22) is utilized. Moreover, every element of $d$ and $r$ can be generated by an autonomous system in the form $\dot{w}_{a}=S_{a} w_{a}, d_{a}=C_{a}^{T} w_{a}$ with appropriate initial values, where $C_{a}=\left[\begin{array}{ll}1 & C_{d}^{T}\end{array}\right]^{T}$. By Lemma 1, if (24) holds, then $\lim _{t \rightarrow \infty} e_{p}(t)=0$ meanwhile keeping $x_{p}(t)$ and $\xi(t)$ bounded. It is easy to see from (22) that both $\tilde{y}_{p}$ and $\dot{\tilde{y}}_{p}$ can be viewed as outputs of a stable system with $e_{p}$ as input. This means that $\tilde{y}_{p}$ and $\dot{\tilde{y}}_{p}$ are bounded if $e_{p}$ is bounded. In addition, $\lim _{t \rightarrow \infty} \tilde{y}_{p}(t)=0$ and $\lim _{t \rightarrow \infty} \dot{\tilde{y}}_{p}(t)=0$.

In most of cases, the controller parameters $L_{1}, L_{2}$ and $L_{3}$ in (23) can be always found. This is shown in the following proposition.

Proposition 1. For any $S=-S^{T}$ without eigenvalues $\pm j$, the parameters

$$
L_{1}=\left[\begin{array}{ll}
1 & C_{d}^{T}
\end{array}\right]^{T}, L_{2}=-\frac{1}{a} C-B-\frac{1}{a} H-\frac{1}{a} K, L_{3}=-\frac{1}{a} L_{1}
$$

can always make max $\operatorname{Re} \lambda\left(A_{a}\right)<0$, where $H=\left[\begin{array}{llll}0 & \varepsilon & 0 & 1\end{array}\right]^{T}$.

Proof. See Appendix $C$.

Remark 5. Proposition 1 in fact implies that, in the presence of any external signal (except for the frequencies at \pm 1 ), the controller (23) with parameters (25) can always make $\lim _{t \rightarrow \infty} y_{p}(t)=r$ and $\lim _{t \rightarrow \infty} \dot{y}_{p}(t)=0$ meanwhile keeping $x_{p}(t)$ and $\xi(t)$ bounded. In other words, the disturbance like $\sin t$ cannot be dealt with, which is consistent with [7]. If the external signal contains the component with frequencies at \pm 1 , then such a frequency component can be chosen not to compensate for, i.e., $S_{a}$ in (23) will not contain eigenvalues $\pm j$.

\section{Stabilized Controller Design for Secondary System}

So far, we have designed the tracking controller for the primary system (17). In this section, we are going to design the stabilized controller for the secondary system (18). It can 
be rewritten as

$$
\begin{aligned}
& \dot{x}_{1, s}=x_{2, s} \\
& \dot{x}_{2, s}=-x_{1, s}+\varepsilon \sin \left(x_{3, s}+r\right)-\varepsilon \sin r+g \\
& \dot{x}_{3, s}=x_{4, s} \\
& \dot{x}_{4, s}=K^{T} x_{s}+v_{s}, x_{s}(0)=0
\end{aligned}
$$

where $g=\varepsilon \sin \left(y_{p}+x_{3, s}\right)-\varepsilon \sin \left(r+x_{3, s}\right)-\varepsilon\left(y_{p}+a \dot{y}_{p}-r\right)$. Our constructive procedure has been inspired by the design in [3]. We will start the controller design procedure from the marginally stable $\left(x_{1, s}, x_{2, s}\right)$-subsystem.

Step 1. Consider the $\left(x_{1, s}, x_{2, s}\right)$-subsystem of (26) with $x_{3, s}$ viewed as the virtual control input. Differentiating the quadratic function $V_{1}=\frac{1}{2}\left(x_{1, s}^{2}+x_{2, s}^{2}\right)$ results in

$$
\dot{V}_{1}=\varepsilon x_{2, s}\left[\sin \left(x_{3, s}+r\right)-\sin r\right]+\varepsilon x_{2, s} g .
$$

Guided by the state-feedback design [10], we introduce the following "Certainty Equivalence" (CE) based virtual controller

$$
x_{3, s}=-b \operatorname{atan} x_{2, s}+x_{3, s}^{\prime}
$$

Then

$$
\begin{aligned}
& \dot{x}_{1, s}=x_{2, s} \\
& \dot{x}_{2, s}=-x_{1, s}+2 \varepsilon \sin \left(\frac{-b \operatorname{atan} x_{2, s}}{2}\right) \cos \left(\frac{-b \operatorname{atan} x_{2, s}+2 r}{2}\right)+g^{\prime}
\end{aligned}
$$

where

$$
g^{\prime}=\varepsilon \sin \left(r-b \operatorname{atan} x_{2, s}+x_{3, s}^{\prime}\right)-\varepsilon \sin \left(r-b \operatorname{atan} x_{2, s}\right)+g .
$$

In order to ensure $\cos \left(\frac{-b \operatorname{atan} x_{2, s}+2 r}{2}\right)>0$, the parameter $b$ is chosen to satisfy $0<b<$ $2(1-2|r| / \pi)$. Since $r \in(-\pi / 2, \pi / 2)$ is a constant, $b$ always exists. The term CE is used here because $x_{3, s}^{\prime}=0$ in (28) makes $\dot{V}_{1}$ in (27) negative semidefinite as $g \equiv 0$.

Step 2. We will apply backstepping to the $\left(x_{3, s}^{\prime}, x_{4, s}\right)$-subsystem and design a nonlinear controller $v_{s}$ to drive $x_{3, s}^{\prime}$ to the origin. By the definition (28), $x_{3, s}^{\prime}=x_{3, s}+b \operatorname{atan} x_{2, s}$. Then the time derivative of the new variable $x_{3, s}^{\prime}$ is

$$
\dot{x}_{3, s}^{\prime}=x_{4, s}+\psi+b \frac{1}{1+x_{2, s}^{2}} g
$$

where $\psi=b \frac{1}{1+x_{2, s}^{2}}\left[-x_{1, s}+\varepsilon \sin \left(x_{3, s}+r\right)-\varepsilon \sin r\right]$. Define a new variable $x_{4, s}^{\prime}$ as follows

$$
x_{4, s}^{\prime}=x_{3, s}^{\prime}+x_{4, s}+\psi \text {. }
$$


Then (31) becomes

$$
\dot{x}_{3, s}^{\prime}=-x_{3, s}^{\prime}+x_{4, s}^{\prime}+b \frac{1}{1+x_{2, s}^{2}} g .
$$

By the definition (32), the time derivative of the new variable $x_{4, s}^{\prime}$ is

$$
\begin{aligned}
\dot{x}_{4, s}^{\prime} & =\dot{x}_{3, s}^{\prime}+\dot{x}_{4, s}+\dot{\psi} \\
& =-x_{3, s}^{\prime}+x_{4, s}^{\prime}+b \frac{1}{1+x_{2, s}^{2}} g+K^{T} x_{s}+v_{s}+\dot{\psi},
\end{aligned}
$$

where

$$
\begin{aligned}
\dot{\psi}= & -2 b x_{2, s} \frac{1}{\left(1+x_{2, s}^{2}\right)^{2}}\left[-x_{1, s}+\varepsilon \sin \left(x_{3, s}+r\right)-\varepsilon \sin r\right] \\
& +b \frac{1}{1+x_{2, s}^{2}}\left[-x_{2, s}+\varepsilon \cos \left(x_{3, s}+r\right) x_{4, s}\right] .
\end{aligned}
$$

Design $v_{s}$ for the secondary system (26) as follows

$$
v_{s}\left(x_{p}, x_{s}, r\right)=x_{3, s}^{\prime}-2 x_{4, s}^{\prime}-K^{T} x_{s}-\dot{\psi}
$$

Then the $\left(x_{3, s}^{\prime}, x_{4, s}^{\prime}\right)$-subsystem becomes

$$
\begin{aligned}
& \dot{x}_{3, s}^{\prime}=-x_{3, s}^{\prime}+x_{4, s}^{\prime}+b \frac{1}{1+x_{2, s}^{2}} g \\
& \dot{x}_{4, s}^{\prime}=-x_{4, s}^{\prime}+b \frac{1}{1+x_{2, s}^{2}} g .
\end{aligned}
$$

It is easy to see that $\lim _{t \rightarrow \infty} x_{3, s}^{\prime}(t)=0$ and $\lim _{t \rightarrow \infty} x_{4, s}^{\prime}(t)=0$ as $\lim _{t \rightarrow \infty} g(t)=0$.

We are now ready to state the theorem for the secondary system.

Theorem 3. Suppose $\lim _{t \rightarrow \infty} y_{p}(t)=r$ and $\lim _{t \rightarrow \infty} \dot{y}_{p}(t)=0$. Let the controller $v_{s}$ for the secondary system (26) be designed as (33), where $0<b<2(1-2|r| / \pi)$. Then $\lim _{t \rightarrow \infty}\left\|x_{s}(t)\right\|=$ 0 meanwhile keeping $x_{s}(t)$ bounded.

Proof. See Appendix D.

\section{E. Controller Synthesis for Original System}

It should be noticed that the controller design above is based on the condition that $x_{p}$ and $x_{s}$ are known as priori. A problem arises that the states $x_{p}$ and $x_{s}$ cannot be measured directly except for $x=x_{p}+x_{s}$. By taking this into account, the following observer is proposed to estimate the states $x_{p}$ and $x_{s}$, which is stated in Theorem 4 .

Theorem 4. Let the observer be designed as follows

$$
\begin{aligned}
& \dot{\hat{x}}_{s}=A \hat{x}_{s}+B v_{s}+\phi(y, \dot{y})-\phi(r, 0) \\
& \hat{x}_{p}=x-\hat{x}_{s}, \hat{x}_{s}(0)=0
\end{aligned}
$$


where $A$ is stable. Then $\hat{x}_{p} \equiv x_{p}$ and $\hat{x}_{s} \equiv x_{s}$.

Proof. Since $x=x_{p}+x_{s}$, we have $y=y_{p}+y_{s}$. Consequently, (35) can be rewritten as

$$
\begin{aligned}
& \dot{\hat{x}}_{s}=A \hat{x}_{s}+B v_{s}+\phi\left(y_{p}+y_{s}, \dot{y}_{p}+\dot{y}_{s}\right)-\phi(r, 0) \\
& \hat{x}_{p}=x-\hat{x}_{s}, \hat{x}_{s}(0)=0 .
\end{aligned}
$$

Subtracting (18) from (36) results in

$$
\dot{\tilde{x}}_{s}=A \tilde{x}_{s}, \tilde{x}_{s}(0)=0
$$

where $\tilde{x}_{s}=\hat{x}_{s}-x_{s}$. Then $\hat{x}_{s} \equiv x_{s}$. Furthermore, with the aid of the relationship $x_{p}=x-x_{s}$, we have $\hat{x}_{p} \equiv x_{p}$.

Remark 6. Unlike traditional observers, the proposed observer can estimate the states of the primary system and the secondary system directly rather than asymptotically or exponentially. This can be explained that, although the initial value $x_{0}$ is unknown, the initial value of either the primary system or the secondary system can be specified exactly, leaving an unknown initial value for the other system. The measurement $x$ and parameters may be inaccurate. In this case, it is expected that small uncertainties lead to $\hat{x}_{p}$ close to $x_{p}$ (or $\hat{x}_{s}$ close to $x_{s}$ ). From (37), a stable matrix $A$ can ensure a small $\tilde{x}_{s}$ in the presence of small uncertainties.

Theorem 5. Suppose that the conditions of Theorems 1-4 hold. Let the controller $u$ in the system (1) be designed as follows

$$
\begin{aligned}
& \dot{\xi}=S_{a} \xi+L_{1}\left[(C+a B)^{T} \hat{x}_{p}-r\right] \\
& u=K^{T} x+v_{p}\left(\xi, \hat{x}_{p}, r\right)+v_{s}\left(\hat{x}_{p}, \hat{x}_{s}, r\right)+\frac{\varepsilon \cos x_{3}}{1-\varepsilon^{2} \cos ^{2} x_{3}} \hat{F}_{d}
\end{aligned}
$$

where $\hat{F}_{d}$ is given by (13), $\hat{x}_{p}$ and $\hat{x}_{s}$ are given by (35), $v_{p}(\cdot)$ is defined in (23), and $v_{s}(\cdot)$ is defined in (33). Then $\lim _{t \rightarrow \infty} y(t)=r$ meanwhile keeping $x$ and $\xi$ bounded.

Proof. Note that the original system (1), the primary system (17) and the secondary system (18) have the relationship: $x=x_{p}+x_{s}$ and $y=y_{p}+y_{s}$. With the controller (38), for the primary system (17), $\lim _{t \rightarrow \infty} y_{p}(t)=r$ meanwhile keeping $x_{p}$ and $\xi$ bounded by Theorem 2. On the other hand, for the secondary system (18), we have $\lim _{t \rightarrow \infty} x_{s}(t)=0$ meanwhile keeping $x_{s}$ bounded on $[0, \infty)$ by Theorem 3 . In addition, Theorem 4 ensures that $\hat{x}_{p} \equiv x_{p}$ and $\hat{x}_{s} \equiv x_{s}$. Therefore, $\lim _{t \rightarrow \infty} y(t)=r$ meanwhile keeping $x$ and $\xi$ bounded. 


\section{NumericAl Simulation}

In the simulation, set $\varepsilon=0.2$ and the initial value $x_{0}=\left[\begin{array}{llll}0 & 0 & 0 & 0\end{array}\right]^{T}$ in (1). The unknown dimensionless disturbance $F_{d}$ is generated by an autonomous LTI system (2) with the parameters as follows

$$
S=\left[\begin{array}{cc}
0 & 2 \\
-2 & 0
\end{array}\right], C_{d}=\left[\begin{array}{ll}
1 & 0
\end{array}\right]^{T}, w(0)=\left[\begin{array}{ll}
0 & 0.02
\end{array}\right]^{T} .
$$

The objective here is to design a controller $u$ such that the output $y(t)=x_{3}(t) \rightarrow r=0.5$ as $t \rightarrow \infty$ meanwhile keeping the other states bounded.

The parameters of the observer (13) are chosen as $l_{1}=l_{2}=10$. In (16), the parameters of $A$ are chosen as $a=1$ and $K=\left[\begin{array}{llll}0 & -\varepsilon & -1 & -2\end{array}\right]^{T}$. Then $\max \operatorname{Re}(\lambda(A))=-0.01<0$. Since matrix $S$ does not possess the eigenvalues $\pm j$, the parameters of the tracking controller (23) of the primary system can be chosen according to Proposition 1 that $L_{1}=\left[\begin{array}{ll}1 & C_{d}^{T}\end{array}\right]^{T}, L_{2}=$ $0_{4 \times 1}$ and $L_{3}=-L_{1}$. These make $A_{a}$ in (24) satisfies $\max \operatorname{Re} \lambda\left(A_{a}\right)=-0.0084<0$. The parameter $b$ of the stabilized controller (33) is chosen as $b=1.5(1-1 / \pi)<2(1-2|r| / \pi)$.

The TORA system (1) is driven by the controller (38) with the parameters above. The evolutions of all states of (1) are shown in Fig.3. As shown, the proposed controller $u$ drives the output $y(t)=x_{3}(t) \rightarrow 0.5$ as $t \rightarrow \infty$, meanwhile keeping the other states bounded.
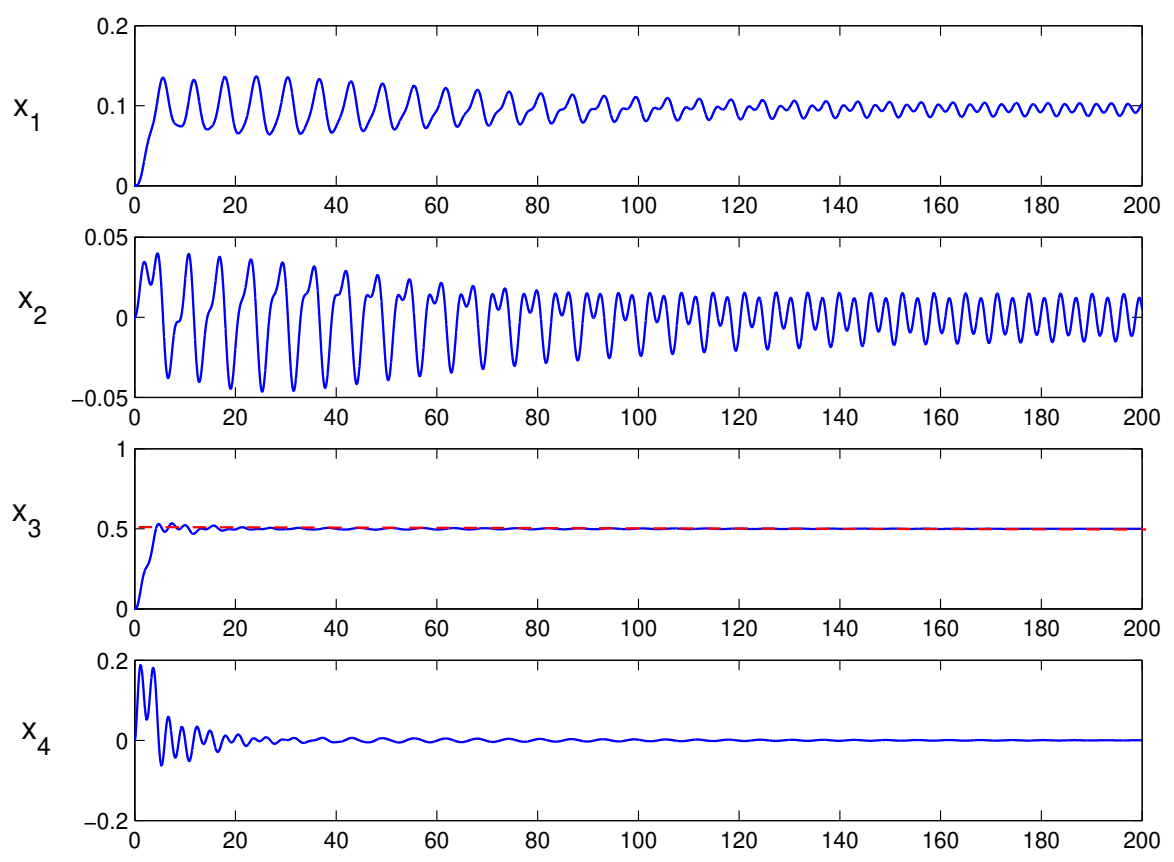

Fig. 3. Evolutions of all states 
Unlike the output regulation theory, the proposed method does not require the regulator equations. If the disturbance $F_{d}$ consists of more frequency components, i.e., $S$ is more complicated, the designed controller above does not need to be changed except for the corresponding $S$ and $C_{d}$. This demonstrates the effectiveness of the proposed control method. For example, we consider that the unknown dimensionless disturbance $F_{d}$ is generated by an autonomous LTI system (2) with the parameters as follows

$$
\begin{aligned}
S & =\operatorname{diag}\left(S_{1}, S_{2}\right), S_{1}=\left[\begin{array}{cc}
0 & 2 \\
-2 & 0
\end{array}\right], S_{2}=\left[\begin{array}{cc}
0 & 1.5 \\
-1.5 & 0
\end{array}\right] \\
C_{d} & =\left[\begin{array}{llll}
1 & 0 & 1 & 0
\end{array}\right]^{T}, w(0)=\left[\begin{array}{llll}
0 & 0.02 & 0 & 0.02
\end{array}\right]^{T} .
\end{aligned}
$$

The controller in the first simulation is still applied to this case except for replacing $S$ and $C_{d}$ (the dimension is changed correspondingly). Driven by the new controller, the evolutions of all states of (1) are shown in Fig.4. As shown, the proposed controller $u$ drives the output $y(t)=x_{3}(t) \rightarrow 0.5$ as $t \rightarrow \infty$, meanwhile keeping the other states bounded.

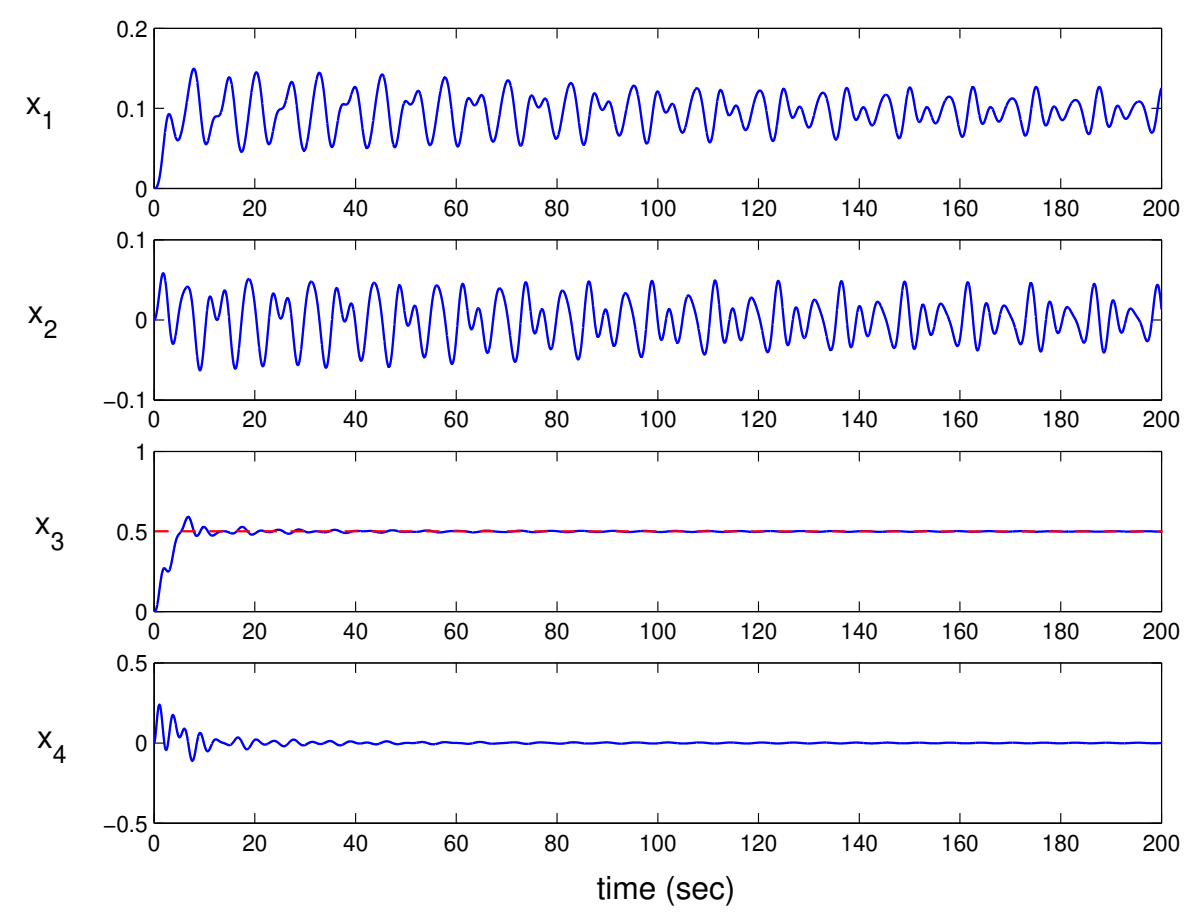

Fig. 4. Evolutions of all states when disturbance is complicated

\section{CONCLUSIONS}

In this paper, the tracking (rejection) problem for rotational position of the TORA was discussed. Our main contribution lies in the presentation of a new decomposition scheme, 
named additive state decomposition, which not only simplifies the controller design but also increases flexibility of the controller design. By the additive state decomposition, the considered system was decomposed into two subsystems in charge of two independent subtasks respectively: an LTI system in charge of a tracking (rejection) subtask, leaving a nonlinear system in charge of a stabilization subtask. Based on the decomposition, the subcontrollers corresponding to two subsystems were designed separately, which increased the flexibility of design. The tracking (rejection) controller was designed by the transfer function method, while the stabilized controller was designed by the backstepping method. This numerical simulation has shown that the designed controller can achieve the objective, moreover, can be changed flexibly according to the model of external signals.

\section{APPENDIX}

\section{A. Proof of Theorem 1}

The disturbance $F_{d} \in \mathbb{R}$ is generated by an autonomous LTI system (2) with an initial value $w(0)$. It can also be generated by the following system

$$
\dot{w}=S w, F_{d}=l_{1} C_{d}^{T} w
$$

with the initial value $\frac{1}{l_{1}} w(0)$. Subtracting (1d) and (39) from (13) results in

$$
\begin{aligned}
& \dot{\tilde{w}}=S \tilde{w}+l_{1} \frac{\varepsilon \cos x_{3}}{1-\varepsilon^{2} \cos ^{2} x_{3}} C_{d} \tilde{x}_{4} \\
& \dot{\tilde{x}}_{4}=-l_{2} \tilde{x}_{4}-l_{1} \frac{\varepsilon \cos x_{3}}{1-\varepsilon^{2} \cos ^{2} x_{3}} C_{d}^{T} \tilde{w}
\end{aligned}
$$

where $l_{1}, l_{2}>0, \tilde{x}_{4} \triangleq \hat{x}_{4}-x_{4}$ and $\tilde{w} \triangleq \hat{w}-w$. Design a Lyapunov function as follows

$$
V_{1}=\frac{1}{2} \tilde{w}^{T} \tilde{w}+\frac{1}{2} \tilde{x}_{4}^{2}
$$

Taking the derivative of $V_{1}$ along (40) results in

$$
\begin{aligned}
\dot{V}_{1} & =\frac{1}{2} \tilde{w}^{T}\left(S+S^{T}\right) \tilde{w}+l_{1} \tilde{w}^{T} \frac{\varepsilon \cos x_{3}}{1-\varepsilon^{2} \cos ^{2} x_{3}} C_{d} \tilde{x}_{4} \\
& -l_{2} \tilde{x}_{4}^{2}-l_{1} \tilde{x}_{4} \frac{\varepsilon \cos x_{3}}{1-\varepsilon^{2} \cos ^{2} x_{3}} C_{d}^{T} \tilde{w} .
\end{aligned}
$$

By Assumption 2, $S+S^{T}=0$. Then the derivative of $V_{1}$ becomes

$$
\dot{V}_{1} \leq-l_{2} \tilde{x}_{4}^{2} \leq 0
$$

Since $l_{2}>0$, from the inequality above, it can be concluded by LaSalle's invariance principle [12] that $\lim _{t \rightarrow \infty} \tilde{x}_{4}(t)=0$ and $\lim _{t \rightarrow \infty} \frac{\varepsilon \cos x_{3}}{1-\varepsilon^{2} \cos ^{2} x_{3}} C_{d}^{T} \tilde{w}(t)=0$. 


\section{B. Proof of Lemma 1}

Before proving Lemma 1, we need the following preliminary result.

Lemma 2. If the pair $\left(A_{z}, B_{z}\right)$ is controllable, then there exists a $C_{0} \in \mathbb{R}^{m}$ such that

$$
C_{0}^{T}\left(s I_{m}-A_{z}\right)^{-1} B_{z}=\frac{1}{\operatorname{det}\left(s I_{m}-A_{z}\right)}
$$

where $A_{z} \in \mathbb{R}^{m \times m}$ and $B_{z} \in \mathbb{R}^{m}$.

Proof. First, we have

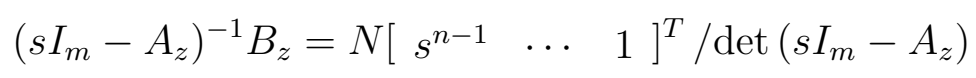

where $N \in \mathbb{R}^{m \times m}$. If the pair $\left(A_{z}, B_{z}\right)$ is controllable, the matrix $N$ is full rank [11]. We can complete this proof by choosing $C_{0}=\left(N^{-1}\right)^{T}\left[\begin{array}{llll}0 & \cdots & 0 & 1\end{array}\right]^{T}$.

With Lemma 2 in hand, we are ready to prove Lemma 1.

i) For the system (20), we have

$$
z(t)=e^{-A_{z} t} z_{0}+\int_{0}^{t} e^{-A_{z}(t-\tau)}\left(d_{a}+\varphi_{a}\right)(\tau) d \tau, t \geq 0
$$

where $d_{a}=\left[\begin{array}{ll}d_{2}^{T} A_{12}^{T} & d_{1}^{T}\end{array}\right]^{T}$ and $\varphi_{a}=\left[\begin{array}{ll}\varphi_{2}^{T} A_{12}^{T} & \varphi_{1}\end{array}\right]^{T}$. Based on the equation above, since $\lambda\left(A_{z}\right)<0$ and $\left\|d_{a}(t)\right\|,\left\|\varphi_{a}(t)\right\|$ are bounded on $[0, \infty)$, it is easy to see that $\left\|z_{1}(t)\right\|$ and $\left\|z_{2}(t)\right\|$ are bounded on $[0, \infty)$.

ii) For the system (20), the Laplace transformation of $z(s)$ is

$$
z(s)=\left(s I-A_{z}\right)^{-1}\left[d_{a}(s)+\varphi_{a}(s)+z_{0}\right] .
$$

Then $z_{1}(s)=C_{a}\left(s I-A_{z}\right)^{-1}\left[d_{a}(s)+\varphi_{a}(s)+z_{0}\right]$, where $C_{a}=\left[\begin{array}{ll}I_{m_{1}} & 0_{m_{1} \times m_{2}}\end{array}\right]^{T}$. The condition $\lambda\left(A_{z}\right)<0$ implies that the pair $\left(S_{z}, A_{12}\right)$ is controllable. Otherwise, for the autonomous system $\dot{z}=A_{z} z$, the variable $z_{1}$ cannot converge to zero as $S_{z}$ is a marginally stable matrix. This contradicts with the condition $\lambda\left(A_{z}\right)<0$. Then by Lemma 1, there exists a $C_{0} \in \mathbb{R}^{m_{1}}$ such that

$$
C_{0}^{T} z_{1}(s)=\frac{1}{\operatorname{det}\left(s I-S_{z}\right)} e_{z}(s)
$$

Then $e_{z}(s)$ can be written as

$$
\begin{aligned}
e_{z}(s) & =\operatorname{det}\left(s I-S_{z}\right) C_{0}^{T} z_{1}(s) \\
& =Q(s) \operatorname{det}\left(s I-S_{z}\right)\left[d_{a}(s)+\varphi_{a}(s)+z_{0}\right] .
\end{aligned}
$$


where $Q(s)=C_{0}^{T} C_{a}\left(s I-A_{z}\right)^{-1} D_{a}$. Since every element of $d_{a}$ can be generated by $\dot{w}_{z}=$ $S_{z} w_{z}, d_{z}=C_{z}^{T} w_{z}$, we have $d_{a}(s)=\left[C_{z}^{T}\left(s I-S_{z}\right)^{-1} w_{z, i}(0)\right]_{\left(m_{1}+m_{2}\right) \times 1}$, where $w_{z, i}(0) \in \mathbb{R}$. Since $\left(s I-S_{z}\right)^{-1}=\frac{1}{\operatorname{det}\left(s I-S_{z}\right)} \operatorname{adj}\left(s I-S_{z}\right), e_{z}(s)$ is further represented as

$$
\begin{aligned}
e_{z}(s)= & Q(s) C_{d}^{T} w_{z}(0)\left[C_{z}^{T} \operatorname{adj}\left(s I-S_{z}\right) w_{z, i}(0)\right]_{\left(m_{1}+m_{2}\right) \times 1} \\
& +\left(s I-A_{z}\right)^{-1}\left[\varphi_{a}(s)+z_{0}\right]
\end{aligned}
$$

Since $\lambda\left(A_{z}\right)<0$ and the order of $A_{z}$ is higher than that of $S_{z}$, moreover $\left\|\varphi_{a}(t)\right\|$ is bounded on $[0, \infty)$ and $\lim _{t \rightarrow \infty}\left\|\varphi_{a}(t)\right\|=0$, for any initial value $w_{z, i}(0)$, we have $\lim _{t \rightarrow \infty} e_{z}(t)=0$ from (41).

\section{Proof of Proposition 1}

If we can prove that the following system

$$
\begin{gathered}
\dot{\xi}=S_{a} \xi+L_{1}(C+a B)^{T} x_{p} \\
\dot{x}_{p}=\left(A+B L_{2}^{T}\right) x_{p}+B L_{3}^{T} \xi
\end{gathered}
$$

is asymptotic stable, then $\operatorname{Re} \lambda\left(A_{a}\right)<0$ holds. Choose a Lyapunov function as follows

$$
V=\frac{1}{2} \xi^{T} \xi+\frac{1}{2} x_{1, p}^{2}+\frac{1}{2} x_{2, p}^{2}+\frac{1}{2} p^{2}
$$

where $p=x_{3, p}+a x_{4, p}=(C+a B)^{T} x_{p}$. With the parameters $L_{1}=\left[\begin{array}{ll}1 & C_{d}^{T}\end{array}\right]^{T}, L_{2}=$ $-\frac{1}{a} C-B-\frac{1}{a} H-K$ and $L_{3}=-\frac{1}{a} L_{1}$, the derivative of $V$ along (42) is

$$
\dot{V}=-p^{2} .
$$

Define $\mathcal{S}=\{x \mid \dot{V}(x)=0\}$, where $x=\left[\begin{array}{ll}\xi^{T} & x_{p}^{T}\end{array}\right]^{T}$. The remaindering work is to prove $\mathcal{S}=\{x \mid x=0\}$. If so, by LaSalle's invariance principle [12], we have $\lim _{t \rightarrow \infty}\|x(t)\|=$ 0 . Therefore, the system (42) with the parameters is globally asymptotically stable. Then $\operatorname{Re} \lambda\left(A_{a}\right)<0$.

Since $\dot{V}=0 \Rightarrow p=0$ and $a>0$, we have $\mathcal{S}=\left\{x \mid x_{3, p}=x_{4, p}=0\right\}$. Let $x$ be a solution belonging to $\mathcal{S}$ identically. Then, from (42), we have

$$
\begin{aligned}
\dot{\xi} & =S_{a} \xi \\
\dot{x}_{1, p} & =x_{2, p}, \dot{x}_{2, p}=-x_{1, p} \\
0 & =-\varepsilon x_{2, p}+L_{3}^{T} \xi
\end{aligned}
$$

From (44), it holds that

$$
x_{2, p} \in \mathcal{S}_{1}=\left\{\left[\begin{array}{ll}
0 & 1
\end{array}\right] \xi \mid \dot{\xi}=\left[\begin{array}{cc}
0 & 1 \\
-1 & 0
\end{array}\right] \xi\right\} .
$$


On the other hand, from (43) and (45), it holds that

$$
x_{2, p} \in \mathcal{S}_{2}=\left\{\frac{1}{\varepsilon} L_{3}^{T} \xi \mid \dot{\xi}=S_{a} \xi\right\}
$$

where matrix $S_{a}$ does not possess eigenvalues $\pm j$ as matrix $S$ does not. Therefore $x_{2, p} \in$ $\mathcal{S}_{1} \cap \mathcal{S}_{2}=\{0\}$ and then $x_{1, p}=0$, namely $\mathcal{S}=\left\{x \mid x_{p}=0\right\}$.

Let $x$ be a solution that belongs identically to $\mathcal{S}$. Then $L_{3}^{T} \xi=0$. Since the pair $\left(C_{d}^{T}, S\right)$ is observable, by the definition $L_{1}=-\frac{1}{a}\left[\begin{array}{ll}1 & C_{d}^{T}\end{array}\right]^{T}$, the pair $\left(L_{3}^{T}, S_{a}\right)$ is observable as well. Consequently, we can conclude that $\xi=0$, namely $\mathcal{S}=\left\{x \mid x=\left[\begin{array}{ll}\xi^{T} & x_{p}^{T}\end{array}\right]^{T}=0\right\}$.

\section{Proof of Theorem 3}

This proof is composed of three parts.

Part 1. $\lim _{t \rightarrow \infty} x_{3, s}^{\prime}(t)=0, \lim _{t \rightarrow \infty} x_{4, s}^{\prime}(t)=0$ and $\lim _{t \rightarrow \infty} g^{\prime}(t)=0$ as $\lim _{t \rightarrow \infty} g(t)=0$. If $\lim _{t \rightarrow \infty} y_{p}(t)=$ $r$ and $\lim _{t \rightarrow \infty} \dot{y}_{p}(t)=0$, then from the definition of $\phi(y, \dot{y})$, we have $\lim _{t \rightarrow \infty} g(t)=0$ no matter what $y_{s}$ is. According to this, it is easy from (34) to see that $\lim _{t \rightarrow \infty} x_{3, s}^{\prime}(t)=0$ and $\lim _{t \rightarrow \infty} x_{4, s}^{\prime}(t)=$ 0 when the controller $v_{s}$ for the secondary system (26) is designed as (33). Then, in (29), $\lim _{t \rightarrow \infty} g^{\prime}(t)=0$.

Part 2. $\lim _{t \rightarrow \infty} x_{1, s}(t)=0$ and $\lim _{t \rightarrow \infty} x_{2, s}(t)=0$. Since $0<b<2(1-2|r| / \pi)$, the derivative $V_{1}$ in (27) negative semidefinite when $g^{\prime}(t) \equiv 0$, namely,

$$
\dot{V}_{1}=2 \varepsilon x_{2, s} \sin \left(\frac{-b \operatorname{atan} x_{2, s}}{2}\right) \cos \left(\frac{-b \operatorname{atan} x_{2, s}+2 r}{2}\right) \leq 0,
$$

where the equality holds at some time instant $t \geq 0$ if and only if $x_{2, s}(t)=0$. By LaSalle's invariance principle [12] that $\lim _{t \rightarrow \infty} x_{1, s}(t)=0$ and $\lim _{t \rightarrow \infty} x_{2, s}(t)=0$ when $g^{\prime}(t) \equiv 0$. Because of the particular structure of $\left(x_{1, s}, x_{2, s}\right)$-subsystem (29), by using [13, Lemma 3.6], one can show that any globally asymptotically stabilizing feedback for $\left(x_{1, s}, x_{2, s}\right)$-subsystem (29) when $g^{\prime}(t) \equiv 0$ achieves global asymptotic stability of $\left(x_{1, s}, x_{2, s}\right)$-subsystem (29) when $\lim _{t \rightarrow \infty} g^{\prime}(t)=0$. Therefore, based on Part 1, $\lim _{t \rightarrow \infty} x_{1, s}(t)=0$ and $\lim _{t \rightarrow \infty} x_{2, s}(t)=0$.

Part 3.Combining the two parts above, we have $\lim _{t \rightarrow \infty}\left\|x_{s}(t)\right\|=0$. For the $\left(x_{1, s}, x_{2, s}\right)$ subsystem and $\left(x_{3, s}^{\prime}, x_{4, s}^{\prime}\right)$-subsystem, $\left\|x_{s}(t)\right\|$ is bounded in any finite time. With the obtained result $\lim _{t \rightarrow \infty}\left\|x_{s}(t)\right\|=0$, we have $\left\|x_{s}(t)\right\|$ is bounded on $[0, \infty)$.

\section{REFERENCES}

[1] Wan C.-J. , Bernstein D.S., Coppola V.T. Global stabilization of the oscillating eccentric rotor. Nonlinear Dynamics 1996; 10: 49-62.

[2] Zhao J., Kanellakopoulos I. Flexible backstepping design for tracking and disturbance attenuation. International Journal of Robust and Nonlinear Control 1998; 8(4-5): 331-348. 
[3] Jiang Z.P., Kanellakopoulos I. Global output-feedback tracking for a benchmark nonlinear system. IEEE Transactions on Automatic Control 2000; 45(5): 1023-1027.

[4] Huang J., Hu G. Control design for the nonlinear benchmark problem via the output regulation method. Journal of Control Theory and Applications 2004; 2(1): 11-19.

[5] Pavlov A., Janssen B., van de Wouw N., Nijmeijer H. Experimental output regulation for a nonlinear benchmark system. IEEE Transactions on Control Systems Technology 2007; 15(4): 786-793.

[6] Fabio C. Output regulation for the TORA benchmark via rotational position feedback. Automatica 2011; 47(3): 584590.

[7] Lan W., Chen B.M., Ding Z. Adaptive estimation and rejection of unknown sinusoidal disturbances through measurement feedback for a class of non-minimum phase non-linear MIMO systems. International Journal of Adaptive Control and Signal Processing 2006; 20(2): 77-97.

[8] Jiang Y., Huang J. Output regulation for a class of weakly minimum phase systems and its application to a nonlinear benchmark system. American Control Conference 2009: 5321-5326.

[9] Y. Liu, W. Wang, and V. Kilic, "Intensity particle flow smc-phd filter for audio speaker tracking," arXiv preprint arXiv:1812.01570, 2018.

[10] Jiang Z.P., Hill D.J., Guo Y. Stabilization and tracking via output feedback for the nonlinear benchmark system. Automatica 1998; 34(7): 907-915.

[11] Cao C., Hovakimyan N. Design and analysis of a novel $\mathcal{L}_{1}$ adaptive control architecture with guaranteed transient performance. IEEE Transactions on Automatic Control 2008; 53(2): 586-591.

[12] Khalil H.K. Nonlinear Systems. Prentice-Hall: Upper Saddle River, NJ, 2002.

[13] Sussmann H.J., Sontag E.D., Yang Y. A general result on the stabilization of linear systems using bounded controls. IEEE Transactions on Automatic Control 1994; 39(12): 2411-2425. 